Selbsthilfegenossenschaften und Nachhaltigkeit

\title{
Durch Teamgründungen zum Arbeitsplatz
}

\section{Lassen sich durch Selbsthilfegenossenschaften nachhaltige Wege zur Sicherung und Erweiterung der Beschäftigung erschließen? Gibt es Instrumente, um die erfolgreiche Gründung von Selbsthilfeunternehmen in genossenschaftlicher Rechtsform zu erleichtern? Der folgende Beitrag gibt Antwort.}

$\mathrm{D}$ Von Burghard Flieger ie innova eG begleitet Erwerbslose bei der Neueinrichtung dauerhafter Arbeitsplätze in Genossenschaften. Zentraler Baustein ist das Durchführen von Fortbildungen in Kooperation mit Bildungsträgern oder sozialen Organisationen, teilweise finanziert vom Arbeitsamt (1). In dieser Zeit arbeiten Erwerbslose eine vorher nur grob skizzierte Geschäftsidee aus und gründen am Ende der Fortbildung eine Genossenschaft, in der sie sich sozialversicherungspflichtige Arbeitsplätze schaffen. Während der Qualifizierung wird das erforderliche Wissen zur Unternehmensführung erworben.

\section{- Was sind Selbsthilfegenossen- schaften?}

Früher wurden wirtschaftliche Selbsthilfe und Genossenschaften oftmals gleichgesetzt. Selbsthilfe beinhaltet das Bestreben, aus eigener Kraft Ziele zu verwirklichen. Sie ist eine Reaktion von Gruppen auf objektive Notlagen oder auf subjektiv als unbefriedigend empfundene Situationen. Diese Situation wollen die Betroffenen in Gruppensolidarität mit dem Ziel einer nachhaltigen Verbesserung der Lebensbedingungen und der Eigenständigkeit ändern. Wesentliches Merkmal von Selbsthilfe ist, dass die eigenen Ressourcen genutzt werden.

Die Überzeugung, sich auf die eigenen Möglichkeiten und auf die Kräfte der Solidarität der Gruppen verlassen zu können, führt oft zur Ausbildung eines größeren Selbstwertgefühls. Auf diese Weise können bei Betroffenen ungeahnte Energien freigesetzt werden. Völlig auf sich gestellt, können sich Betroffene überfordert fühlen. Zielgruppen von Selbsthilfe sind in der Regel Personengruppen, die Ausgrenzung und Benachteiligung erfahren. Insofern bringt Hilfe zur Selbsthilfe als begrenzte Förderung von außen die positiven Energien der Selbsthilfe in Form von Empowerment erst zum Tragen (2).
Unter einer Selbsthilfegenossenschaft ist der Zusammenschluss einer Gruppe von Menschen unterhalb der Armutsgrenze zu verstehen, die Ausgrenzung und Benachteiligung erfahren. Sie helfen sich selbst über wirtschaftliche Aktivitäten in einer Organisation, die nach den genossenschaftlichen Prinzipien des Förder-, Identitäts-, Demokratie- und Solidaritätsprinzips strukturiert wird. Bis eine Selbsthilfegenossenschaft auf eigenen Beinen steht, kann sie über Beratung, Qualifizierung, Betreuung, Zuschüsse bei der Finanzierung etc Hilfe von außen erfahren. Diese Hilfe sollte so angelegt sein, dass sie die Eigeninitiative und Selbstverantwortung der Genossenschaftsmitglieder stärkt.

Förderwirtschaftliches Agieren ist ein entscheidendes Charakteristikum genossenschaftlicher Unternehmen und damit auch von Selbsthilfegenossenschaften. Insofern steht dieses Charakteristikum häufig im Vordergrund, um die Abgrenzung gegenüber anderen Unternehmensformen zu verdeutlichen. Es besagt: Nicht die Verwertung von Kapital und das Erwirtschaften von Gewinn soll Hauptzweck einer Genossenschaft sein, sondern die Förderung der Mitglieder in dem Geschäftsfeld, in dem das Unternehmen angesiedelt ist. Hierin liegt der materielle Kern der Nachhaltigkeit von Selbsthilfegenossenschaften, indem die Schaffung von Arbeitsplätzen im Förderauftrag formuliert und umgesetzt wird.

Das empirisch am leichtesten überprüfbare genossenschaftliche Prinzip und somit das eindeutigste Erkennungsmerkmal stellt das Identitätsprinzip dar. Es besagt: Zwei Rollen, die sich sonst am Markt gegenüberstehen, fallen in der Personengruppe der Genossenschaftler zusammen. Bei der Wohnungsbaugenossenschaft sind es Mieter und Vermieter, in der Selbsthilfegenossenschaft, sind die Helfenden und die sich selbst Helfenden identisch. Sie sind Anbieter (Arbeitgeber) und Nutzer (Arbeitnehmer) der Selbsthilfeleistung beispielsweise in Form von Arbeitsplätzen.
Mit dem Demokratieprinzip ,ein Mensch eine Stimme" werden Genossenschaften am stärksten verbunden. Damit ist zugleich der strukturell-organisatorische Aspekt der Nachhaltigkeit verbunden, der allzu oft bei der Verfolgung ökologischer Aspekte und damit auch bei Gründung von Umweltunternehmen vernachlässigt wird.

Als letztes Prinzip soll das Solidaritätsprinzip genannt werden, auch wenn es das umstrittenste ist. Bekannter ist der Begriff Genossenschaftsgeist. Mit diesem Prinzip wird das Thema Unternehmenskultur in besonderem Maße tangiert, weil es um die Ausprägung genossenschaftsspezifischer Werte, Einstellungen und Verhaltensweisen geht.

\section{Chancenverbesserung für Benachteiligte}

Die Gründung von Selbsthilfegenossenschaften ist unter arbeitsmarktpolitischem Blickwinkel als Ziel und Mittel zugleich zu sehen. Ziel sind sie, weil Benachteiligte durch die Integration in Genossenschaften Gleichberechtigung und Partizipation erleben. Der „Mittelcharakter“ der Genossenschaft kommt dadurch zum Ausdruck, dass in keiner anderen Unternehmensform durch das Selbstverständnis und die Strukturen so viele Chanceneröffnungen für Empowerment bestehen: Bei den arbeitsmarktpolitischen Zielgruppen werden die individuellen Fähigkeiten und Motivationen über die Einbindung mehr als bei anderen Organisationsformen mobilisiert.

Hintergrund hierfür sind die mit dieser Organisation verbundenen Partizipationsmöglichkeiten. Praxisgerecht konzipierte Beteiligungssysteme steigern nicht nur die Produktivität, die Wettbewerbsfähigkeit und die Rentabilität der Unternehmen, sie verbessern gleichzeitig auch die Mitwirkung der Mitarbeiter, die Arbeitsqualität und können zu einem stärkeren sozialen Zusammenhalt beitragen. Als Gründe dafür werden von einer Studie des Instituts für Arbeitsmarkt und Berufsforschung (IAB) mehrere Wirkungsmechanismen von materieller und immaterieller Partizipation herausgestellt (3):

- höhere Bereitschaft der Beschäftigten zur eigenen Qualifizierung;

- größere Bereitschaft sparsam mit den betrieblichen Ressourcen umzugehen;

- Engagement für Verbesserungen der Arbeitsorganisation;

- zunehmende Interessenidentität bei den Beschäftigten und damit verbesserte Zusammenarbeit;

- sinkende Mitarbeiterfluktuation; 


\section{Abbildung 1: Genossenschafflicher Projektentwicklung}

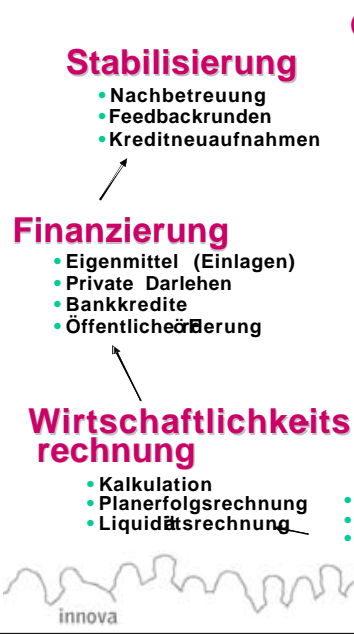

Gescläftsidee Bausteine

Produkt/Markt für die Gründung

Kunden
Konkurrenz
von Selbsthilfe -

$\begin{aligned} & \text { Konkurrenz } \\ & \text { Marketing }\end{aligned}$ venossenschaften
gen Selbsthilfe -

Qualifizierung

Lehrönge

Tagesseminare

Workshops /

Gruppenbetreuung

- Mitglieder

-Arbeitsorganisation

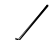

Rechtsform

Genossenschaftssatzung

Entscheidungszuordnung

\section{ran}

Quelle: eigene Darstellung

- Rückgang an Fehlzeiten;

- Verbesserung des Betriebsklimas;

- verbesserter Informationsfluss.

\section{- Projektentwicklung}

Doch Selbsthilfegenossenschaften sind keine Selbstläufer und auch kein Allheilmittel für arbeitsmarktpolitische Probleme. Weder werden konjunkturelle noch makroökonomische Bedingungen durch einzelbetriebliche Gründungen wesentlich beeinflusst. Hinzu kommt, dass die GründerInnen der Selbsthilfegenossenschaften zu den am Arbeitmarkt Benachteiligten gehören. Es lassen sich sieben Bausteine festhalten, die besonders wichtig sind, um Selbsthilfegenossenschaften zu gründen und zu gefestigten Unternehmen weiterzuentwickeln.

Zur Strukturierung der Bausteine wurde von der innova eG ein Vierphasenschema entwickelt. Die Orientierungsphase dient zur Klärung, ob ein Gruppenunternehmen in der Rechtsform der Genossenschaft verfolgt werden soll. Als Erfolg wird hier gesehen, wenn am Ende der Orientierungsphase eine sachbezogene fundierte Entscheidung fällt. Diese kann gegebenenfalls auch negativ gegenüber einer Unternehmensgründung sein. Im Mittelpunkt der zweiten Phase, der Planungsund Konzeptphase, steht die Fundierung der Geschäftsidee und die Prüfung ihrer wirtschaftlichen Tragfähigkeit. Diese Phase ist erfolgreich abgeschlossen, wenn am Ende ein genossenschaftliches Unternehmenskonzept vorliegt, das Externen wie dem genossenschaftlichen Prüfungsverband oder Finanziers zur Begutachtung gegeben werden kann.

Die Gründungsphase selbst fällt relativ kurz aus. Sie wird aber als Phase zusätzlich herausgestellt, weil es hier ,zum Schwur“ kommt. Wichtige Ent- scheidungen, auf die über einen längeren Zeitraum hingearbeitet wurde, werden verwirklicht. Im Idealfall wird die Gruppe mit der Gründung in die Selbständigkeit entlassen. Die bisherigen Erfahrungen zeigen aber, dass fast immer ein weiterhin hoher Klärungs- und Betreuungsbedarf besteht. Ohne Stabilisierungshilfen in der Aufbausituation sind die GründerInnen oftmals schnell überfordert. Verschiedene Formen der Unterstïtzung sind nachbereitend erforderlich und werden auch im begrenzten Rahmen von innova geleistet. Typische Bausteine in dieser Phase sind nachhaltige Stabilisierung und Verankerung des Unternehmens am Markt, Aufbau einer Unterstützungsstruktur, Nachfolge- und Ergänzungsfinanzierungen, Modifizierung und Ausweitung der Geschäftsidee.

\section{Anmerkungen}

(1) Flieger, B.: Qualifizieren als Hilfe zur Selbsthilfe. In: Flieger, B. (Hrsg.): Sozialgenossenschaften - Wege zu mehr Beschüftigung, bürgerschaftlichem Engagement und Arbeitsformen der Zukunft. Neu-Ulm 2003, S. 133-156. (2) Elsen, S.: Lässt sich Gemeinwesenökonomie durch Genossenschaften aktivieren? Chancen für Empowerment. In: Flieger, B: Sozialgenossenschaften, a.a.0., S. $57 \mathrm{ff}$.

(3) Iris Möller: Mitarbeiterbeteiligung: Ein Weg zu höherer Produktivität. IAB-Kurzbericht Nr. 9. Nürnberg 2002.

\section{Der Autor}

Dr. Burghard Flieger ist wissenschaftlicher Leiter und Vorstand der innova eG

Kontakt: innova eG, Konstantinstr. 12, 04315 Leipzig. Tel. 0341-6810985, E-Mail: info@innova-eg.de, www.innova-eg.de. 
(c) 20I0 Authors; licensee IÖW and oekom verlag. This is an article distributed under the terms of the Creative Commons Attribution Non-Commercial No Derivates License (http://creativecommons.org/licenses/by-nc-nd/3.o/), which permits unrestricted use, distribution, and reproduction in any medium, provided the original work is properly cited. 